\title{
FIRST REPORT OF THE PALAU BIRD RECORDS COMMITTEE
}

DEMEI OTOBED, ALAN R. OLSEN ${ }^{\dagger}$, and MILANG EBERDONG, Belau National Museum, P.O. Box 666, Koror, Palau 96940

HEATHER KETEBENGANG, Palau Conservation Society, P.O. Box 1181, Koror, Palau 96940; palaubirdrecords@gmail.com

MANDY T. ETPISON, Etpison Museum, P.O. Box 7049, Koror, Palau 96940

H. DOUGLAS PRATT, 1205 Selwyn Lane, Cary, North Carolina 27511

GLENN H. MCKINLAY, C/55 Albert Road, Devonport, Auckland 0624, New Zealand

GARY J. WILES, 521 Rogers St. SW, Olympia, Washington 98502

ERIC A. VANDERWERF, Pacific Rim Conservation, P.O. Box 61827, Honolulu, Hawaii 96839

MARK O'BRIEN, BirdLife International Pacific Regional Office, 10 MacGregor Road, Suva, Fiji

RON LEIDICH, Planet Blue Kayak Tours, P.O. Box 7076, Koror, Palau 96940

UMAI BASILIUS and YALAP YALAP, Palau Conservation Society, P.O. Box 1181, Koror, Palau 96940

ABSTRACT: After compiling a historical list of 158 species of birds known to occur in Palau, the Palau Bird Records Committee accepted 10 first records of new occurrences of bird species: the Common Pochard (Aythya ferina), Black-faced Spoonbill (Platalea minor), Chinese Pond Heron (Ardeola bacchus), White-breasted Waterhen (Amaurornis phoenicurus), Eurasian Curlew (Numenius arquata), Gull-billed Tern (Gelochelidon nilotica), Channel-billed Cuckoo (Scythrops novaehollandiae), Ruddy Kingfisher (Halcyon coromanda), Common Kingfisher (Alcedo atthis), and Isabelline Wheatear (Oenanthe isabellina). These additions bring Palau's total list of accepted species to 168. We report Palau's second records of the Broad-billed Sandpiper (Calidris falcinellus), Chestnut-winged Cuckoo (Clamator coromandus), Channelbilled Cuckoo, White-throated Needletail (Hirundapus caudacutus) and Oriental Reed Warbler (Acrocephalus orientalis). This report concludes with a current list of the bird species known from Palau.

This is the first report of the Palau Bird Records Committee (PBRC). Situated in the western equatorial Pacific and within the East Asian/Australasian Flyway, Palau has the richest bird diversity of any island group in Micronesia (Wiles 2005). The board of trustees of Belau National Museum established the PBRC on 8 December 2014 as a committee of the museum's National Program for Monitoring Forest and Coastal Birds. The geographic scope of the committee is all islands that are part of the Republic of Palau and the waters within 200 nautical miles of the coast of the Palau Islands. The mission of the committee is to review noteworthy reports of field observations of Palau's birds for the purpose of maintaining an authoritative Palau Islands

\footnotetext{
${ }^{\dagger}$ Alan R. Olsen, a passionate birder whose dedication to chronicling Palau's birds will be forever remembered and appreciated, died suddenly and unexpectedly on 24 June 2018. His labor of love resulted in the attainment of a number of significant forest conservation milestones. Palau is much richer in knowledge for having known and considered Alan as one of our own.
} 
bird list and building a broader understanding of the avifauna of Palau. The $\mathrm{PBRC}$ is the first, and so far only, bird records committee in Micronesia and the second such committee in the tropical Pacific (Hawaii has the other).

Establishment of the PBRC was motivated by the entry into force of the United Nations Convention on the Conservation of Migratory Species of Wild Animals in the Republic of Palau and the designation of the Northern Peleliu Lkes in Palau as a globally Important Bird Area and regionally (Oceania) significant site for migratory shorebirds (BirdLife International 2018). The charge to review "noteworthy" reports is purposefully flexible to permit verification of sightings of banded birds, clarification of which species are passage migrants versus winter visitors, and monitoring of other developments in Palau's sector of the East Asian/Australasian Flyway.

\section{MEMBERSHIP AND PROCEDURES}

The PBRC's membership and procedures follow bylaws established by the board of trustees of Belau National Museum. The president of the board of trustees (Otobed) is ex officio chair of the PBRC. Other committee members serve at the discretion of the board of trustees. The committee consists of a records panel, a report review panel, and an advisory group. Membership in the records panel is restricted to residents of Palau who are affiliated with Belau National Museum, Palau Conservation Society, or other local conservation organizations. Membership in the report review panel is open to resident and off-island experts in the identification of birds, especially birds that are likely to appear in Palau. Membership in the advisory group is limited to residents of Palau. The records panel (Olsen, Eberdong, and Ketebengang) receives reports of bird observations, administers the review of the reports, and maintains an up-to-date list of the birds of Palau. The report review panel (Etpison, McKinlay, O'Brien, Pratt, Wiles, and VanderWerf) reviews all reports received by the records panel, recommending acceptance or rejection of new records; reports of the occurrence (or extirpation) of a species; and other noteworthy reports. The advisory group (Leidich, Basilius, and Yalap) coordinates with organizations and initiatives that share an interest in an authoritative checklist of Palau birds. The committee's review procedures reflect Palau's traditional consensus-based decision-making process. When the report review panel reaches consensus on the acceptance or rejection of a report, its decision is forwarded through the records panel to the chair for final approval/disapproval as an official decision of the committee. The chair's approval of a new record and subsequent publication in one of the committee's reports in Western Birds or other peer-reviewed journal places that species on the official Palau Islands bird list maintained by the records panel (see Table 2).

\section{HISTORICAL LIST OF PALAU'S BIRDS}

In his monograph on the avifauna of Micronesia, Baker (1951) provided accounts of 92 species from Palau based on field observations (Baker 1948), museum specimens, and a thorough critical review of the literature from the mid-19 $9^{\text {th }}$ century, when the first collectors for European museums 
visited Palau, through the mid-20 th $c e n t u r y$. Baker examined 791 museum specimens from Palau representing 63 species, including 221 specimens (47 species) that he collected and 570 additional specimens in the American Museum of Natural History, Museum of Comparative Zoology at Harvard University, University of Kansas Museum of Natural History, and U.S. National Museum of Natural History (USNM). He included accounts of 17 additional species on the basis of credible identifications and collection data provided by German ornithologists from specimens in the Godeffroy Museum in Hamburg (Hartlaub 1868, Hartlaub and Finsch 1868a, 1868b, 1872, Finsch 1875) and by Japanese ornithologists from specimens in their collections (Kuroda 1922a, 1922b, Momiyama 1922, Hachisuka et al. 1942). Baker did not mention contemporary Palau specimens in the Museum of Vertebrate Zoology, University of California, Berkeley (Marshall 1951) and the Yale Peabody Museum (YPM) (Ripley 1951), though the species represented by these specimens were included in Baker's monograph on the basis of specimens in the other museums. Baker accepted 10 additional migratory species listed by Mayr (1945), mentioned Ripley's (1948) report of the Oriental Darter (Anhinga melanogaster), and included the Far Eastern Curlew Numenius madagascariensis on the basis of his own party's sightings only.

Wiles (2005) compiled an annotated checklist of 148 species for Palau that accepted 90 of the 92 species itemized by Baker (1951). Wiles purged the Scaly-breasted Munia (Lonchura punctulata) as extirpated in Palau (Pyle and Engbring 1985) and the Oriental Darter as a record of questionable accuracy. He added 58 species on the basis of a combination of field observations (Wiles and Conry 1990, Wiles et al. 2000, 2004), a full review of Baker's $(1948,1951)$ monographs and references, and critical reviews of reports by field ornithologists who visited or lived in Palau after Baker (Marshall 1951, Ripley 1951, Dixon and Starrett 1952, Bruyns 1964, Owen 1977a, 1977b, Pratt et al. 1980, 1987, Engbring and Owen 1981, Engbring 1983, 1988, Rauzon 1988). Wiles found three anomalous reports that he subsequently verified from museum specimens, of the Black-headed Bunting (Emberiza melanocephala; USNM 336692, Owen 1977a), Pale-vented Bush-Hen (Amaurornis moluccana; USNM 582225, Engbring and Owen 1981), and Rufous Hawk-Cuckoo (Hierococcyx hyperythrus; YPM 12390, Ripley 1951), and a report of the Rainbow Bee-eater (Merops ornatus), which he verified from the observer's field notes (J. Engbring pers. comm.). Pratt and Etpison (2008) discussed all of the species listed for Palau by Wiles (2005) with recent photos and illustrations of 129 species.

The committee's first action (tracking number P001) was to approve a historical list of 158 species of Palau birds, including 146 listed by Wiles (2005), 10 (Table 1) subsequently reported by VanderWerf et al. (2006) and Pratt et al. (2010), and two additional species of Caprimulgus nightjars as a result of a taxonomic revision (Gill and Donsker 2018). Two of the species from Wiles' checklist were deleted: the nightjar Caprimulgus indicus, as a result of its taxonomic breakup into three species, and the Asian House Martin (Delichon dasypus), because of the equivocal nature of the evidence (Pratt et al. 2010). 
Table 1 Species First Recorded from Palau 2004-2009.

\begin{tabular}{|c|c|c|c|c|}
\hline English name & Scientific name & Date & Locality & Source $e^{a}$ \\
\hline Glossy Ibis & Plegadis falcinellus & 17 Jun 2009 & Koror & $P$ \\
\hline Gray Heron & Ardea cinerea & 3 May 2005 & Peleliu & $\mathrm{V}$ \\
\hline Common Snipe & Gallinago gallinago & 17 Nov 2008 & Koror & $\mathrm{P}$ \\
\hline $\begin{array}{l}\text { Red-necked } \\
\text { Phalarope }\end{array}$ & Phalaropus lobatus & 10 Oct 2006 & Koror & $P$ \\
\hline Green Sandpiper & Tringa ochropus & Oct 2006 & Koror & $\mathrm{P}$ \\
\hline Brown Hawk-Owl & Ninox scutulata & Nov 2004 & Helen Reef & $\mathrm{P}$ \\
\hline $\begin{array}{l}\text { White-throated } \\
\text { Needletail }\end{array}$ & $\begin{array}{l}\text { Hirundapus } \\
\text { caudacutus }\end{array}$ & 13 Apr 2004 & Peleliu & $\mathrm{P}$ \\
\hline $\begin{array}{l}\text { Oriental Reed } \\
\text { Warbler }\end{array}$ & $\begin{array}{c}\text { Acrocephalus } \\
\text { orientalis }\end{array}$ & 11 Apr 2007 & Koror & $\mathrm{P}$ \\
\hline Scaly Thrush & Zoothera dauma & Jan 2006 & Koror & $\mathrm{P}$ \\
\hline Blyth's Pipit ${ }^{b}$ & Anthus godlewskii & 23 Apr 2005 & Koror & V \\
\hline
\end{tabular}

${ }^{a} \mathrm{~V}$, VanderWerf et al. (2006) ; P, Pratt et al. (2010).

${ }^{b}$ Identification corrected; see Other Committee Actions.

\section{RECORDS ACCEPTED BY THE COMMITTEE}

The committee accepted 15 of the 18 reports of bird observations it received as of 31 December 2016 as first or second records for Palau, as itemized below. The data for each species include its English name, scientific name, the committee's tracking number, number of birds constituting the record, their age and/or sex if evident (figure number), inclusive dates of occurrence, locality, responsible party, and a brief summary of other relevant information. The locality "Northern Peleliu Lkes Important Bird Area" is spelled correctly. "Lkes" is Palauan for "intertidal sand flats."

Common Pochard Aythya ferina. P006. Adult male (Figure 1a), 19 Nov 2014, Melekeok (Lake Ngardok Nature Reserve), Heather Ketebengang. Pratt et al. (2010) considered prior reports of the species in Palau to be equivocal. The only other Micronesian records are from Guam and the Northern Marianas (Wiles 2005).

Black-faced Spoonbill Platalea minor. P003. One bird (Figure 1b), 8 Dec 2013 to 15 Mar 2014, Peleliu (Northern Peleliu Lkes Important Bird Area), Glenn McKinlay. A separate report (McKinlay 2015) described the bird in detail sufficient to distinguish it from congeners. The Black-faced Spoonbill is an endangered species (IUCN 2017) with a restricted range. It migrates from breeding grounds on the west coast of the Korean peninsula, northeastern China, and northeastern Russia to winter in southern China, Taiwan, Vietnam, and southern Japan (Gill and Donsker 2018). It has not been previously reported from Micronesia.

Chinese Pond Heron Ardeola bacchus. P010. One adult in alternate plumage (Figure 1c), 12-14 May 2014, Koror (Malakal sewage treatment plant), Glenn McKinlay. Pratt et al. (2010) reported a heron of the genus Ardeola photographed 12-23 Dec 2008 at the Koror landfill by Heather Ketebengang. Although the photos showing a bird in basic plumage were "strongly suggestive" of A. bacchus, Pratt et al. (2010) concluded that species identification was equivocal because the basic plumage of $A$. bacchus is indistinguishable from that of the Javan Pond Heron (A. speciosa). Wiles et al. $(2000,2004)$ handled earlier reports of Ardeola in basic plumage from Guam and the Northern Marianas in similar fashion. This is the first unequivocal record for 
Table 2 Palau Islands Bird List.

\begin{tabular}{|c|c|c|c|}
\hline Family & Genus/species $^{a}$ & English name $e^{a}$ & Status ${ }^{b}$ \\
\hline \multirow[t]{7}{*}{ Anatidae } & Spatula querquedula & Garganey & M \\
\hline & Mareca penelope & Eurasian Wigeon & M \\
\hline & Anas superciliosa & Pacific Black Duck & $\mathrm{R}$ \\
\hline & Anas acuta & Northern Pintail & M \\
\hline & Anas crecca & Eurasian Teal & M \\
\hline & Aythya ferina & Common Pochard & $*$ \\
\hline & Aythya fuligula & Tufted Duck & M \\
\hline Megapodiidae & Megapodius laperouse & Micronesian Megapode & $\mathrm{R}$ \\
\hline Phasianidae & Gallus gallus & Red Junglefowl & I \\
\hline Hydrobatidae & Oceanodroma matsudairae & Matsudaira's Storm Petrel & $\mathrm{P}$ \\
\hline \multirow[t]{5}{*}{ Procellariidae } & Pterodroma solandri & Providence Petrel & $\mathrm{P}$ \\
\hline & Calonectris leucomelas & Streaked Shearwater & $\mathrm{P}$ \\
\hline & Ardenna pacifica & Wedge-tailed Shearwater & $\mathrm{S}$ \\
\hline & Puffinus bailloni & Tropical Shearwater & $\mathrm{R}$ \\
\hline & Bulweria bulwerii & Bulwer's Petrel & $\mathrm{P}$ \\
\hline \multirow[t]{2}{*}{ Phaethontidae } & Phaethon rubricauda & Red-tailed Tropicbird & $\mathrm{R}$ \\
\hline & Phaethon lepturus & White-tailed Tropicbird & $\mathrm{R}$ \\
\hline \multirow[t]{2}{*}{ Threskiornithidae } & Plegadis falcinellus & Glossy Ibis & M \\
\hline & Platalea minor & Black-faced Spoonbill & $*$ \\
\hline \multirow[t]{14}{*}{ Ardeidae } & Ixobrychus sinensis & Yellow Bittern & $\mathrm{R}$ \\
\hline & Ixobrychus eurhythmus & Von Schrenck's Bittern & $\mathrm{V}$ \\
\hline & Gorsachius goisagi & Japanese Night Heron & $\mathrm{V}$ \\
\hline & Gorsachius melanolophus & Malayan Night Heron & $\mathrm{V}$ \\
\hline & Nycticorax nycticorax & Black-crowned Night Heron & M \\
\hline & Nycticorax caledonicus & Rufous Night Heron & $\mathrm{R}$ \\
\hline & Butorides striata & Striated Heron & M \\
\hline & Ardeola bacchus & Chinese Pond Heron & $*$ \\
\hline & Bubulcus coromandus & Eastern Cattle Egret & M \\
\hline & Ardea cinerea & Gray Heron & M \\
\hline & Ardea alba & Great Egret & M \\
\hline & Ardea intermedia & Intermediate Egret & M \\
\hline & Egretta garzetta & Little Egret & M \\
\hline & Egretta sacra & Pacific Reef Heron & $\mathrm{R}$ \\
\hline Pelecanidae & Pelecanus conspicillatus & Australian Pelican & V \\
\hline \multirow[t]{2}{*}{ Fregatidae } & Fregata minor & Great Frigatebird & $\mathrm{R}$ \\
\hline & Fregata ariel & Lesser Frigatebird & $\mathrm{S}$ \\
\hline \multirow[t]{3}{*}{ Sulidae } & Sula dactylatra & Masked Booby & $\mathrm{S}$ \\
\hline & Sula sula & Red-footed Booby & $\mathrm{R}$ \\
\hline & Sula leucogaster & Brown Booby & $\mathrm{R}$ \\
\hline \multirow[t]{2}{*}{ Phalacrocoracidae } & Microcarbo melanoleucos & Little Pied Cormorant & $\mathrm{R}$ \\
\hline & Phalacrocorax sulcirostris & Little Black Cormorant & $\mathrm{V}$ \\
\hline Pandionidae & Pandion haliaetus & Western Osprey & M \\
\hline \multirow[t]{4}{*}{ Accipitridae } & Accipiter soloensis & Chinese Sparrowhawk & M \\
\hline & Milvus migrans & Black Kite & (M) \\
\hline & Haliastur indus & Brahminy Kite & (V) \\
\hline & Butastur indicus & Gray-faced Buzzard & (V) \\
\hline \multirow[t]{4}{*}{ Rallidae } & Rallina fasciata & Red-legged Crake & V \\
\hline & Rallina eurizonoides & Slaty-legged Crake & $\mathrm{R}$ \\
\hline & Gallirallus philippensis & Buff-banded Rail & $\mathrm{R}$ \\
\hline & Amaurornis moluccana & Pale-vented Bush-hen & V \\
\hline
\end{tabular}


Table 2 (continued).

\begin{tabular}{|c|c|c|c|}
\hline Family & Genus/species ${ }^{a}$ & English name $e^{a}$ & Status ${ }^{b}$ \\
\hline & Amaurornis phoenicurus & White-breasted Waterhen & $*$ \\
\hline & Porzana cinerea & White-browed Crake & $\mathrm{R}$ \\
\hline & Porphyrio melanotus & Australasian Swamphen & $\mathrm{R}$ \\
\hline & Gallinula chloropus & Common Moorhen & $\mathrm{R}$ \\
\hline Recurvirostridae & Himantopus himantopus & Black-winged Stilt & M \\
\hline \multirow{9}{*}{ Charadriidae } & Erythrogonys cinctus & Red-kneed Dotterel & $\mathrm{V}$ \\
\hline & Pluvialis fulva & Pacific Golden Plover & M \\
\hline & Pluvialis squatarola & Gray Plover & M \\
\hline & Charadrius hiaticula & Common Ringed Plover & M \\
\hline & Charadrius dubius & Little Ringed Plover & M \\
\hline & Charadrius alexandrinus & Kentish Plover & M \\
\hline & Charadrius mongolus & Lesser Sand Plover & M \\
\hline & Charadrius leschenaultii & Greater Sand Plover & M \\
\hline & Charadrius veredus & Oriental Plover & $\mathrm{V}$ \\
\hline \multirow[t]{30}{*}{ Scolopacidae } & Numenius phaeopus & Whimbrel & M \\
\hline & Numenius minutus & Little Curlew & M \\
\hline & Numenius madagascariensis & Far Eastern Curlew & M \\
\hline & Numenius arquata & Eurasian Curlew & $*$ \\
\hline & Limosa lapponica & Bar-tailed Godwit & M \\
\hline & Limosa limosa & Black-tailed Godwit & M \\
\hline & Arenaria interpres & Ruddy Turnstone & M \\
\hline & Calidris tenuirostris & Great Knot & M \\
\hline & Calidris canutus & Red Knot & V \\
\hline & Calidris pugnax & Ruff & M \\
\hline & Calidris falcinellus & Broad-billed Sandpiper & V \\
\hline & Calidris acuminata & Sharp-tailed Sandpiper & M \\
\hline & Calidris ferruginea & Curlew Sandpiper & M \\
\hline & Calidris subminuta & Long-toed Stint & M \\
\hline & Calidris ruficollis & Red-necked Stint & M \\
\hline & Calidris alba & Sanderling & M \\
\hline & Calidris alpina & Dunlin & M \\
\hline & Calidris melanotos & Pectoral Sandpiper & M \\
\hline & Gallinago megala & Swinhoe's Snipe & M \\
\hline & Gallinago gallinago & Common Snipe & M \\
\hline & Xenus cinereus & Terek Sandpiper & M \\
\hline & Phalaropus lobatus & Red-necked Phalarope & V \\
\hline & Actitis hypoleucos & Common Sandpiper & M \\
\hline & Tringa ochropus & Green Sandpiper & V \\
\hline & Tringa incana & Wandering Tattler & M \\
\hline & Tringa brevipes & Gray-tailed Tattler & M \\
\hline & Tringa totanus & Common Redshank & M \\
\hline & Tringa stagnatilis & Marsh Sandpiper & M \\
\hline & Tringa glareola & Wood Sandpiper & M \\
\hline & Tringa nebularia & Common Greenshank & M \\
\hline Glareolidae & Glareola maldivarum & Oriental Pratincole & M \\
\hline \multirow[t]{6}{*}{ Laridae } & Anous stolidus & Brown Noddy & $\mathrm{R}$ \\
\hline & Anous minutus & Black Noddy & $\mathrm{R}$ \\
\hline & Gygis alba & White Tern & $\mathrm{R}$ \\
\hline & Chroicocephalus ridibundus & Black-headed Gull & M \\
\hline & Gelochelidon nilotica & Gull-billed Tern & $*$ \\
\hline & Thalasseus bergii & Greater Crested Tern & $\mathrm{R}$ \\
\hline
\end{tabular}


Table 2 (continued).

\begin{tabular}{|c|c|c|c|}
\hline Family & Genus/species ${ }^{a}$ & English name $e^{a}$ & Status $^{b}$ \\
\hline & Sternula albifrons & Little Tern & $\mathrm{M}$ \\
\hline & Onychoprion lunatus & Spectacled Tern & S \\
\hline & Onychoprion anaethetus & Bridled Tern & $\mathrm{R}$ \\
\hline & Onychoprion fuscatus & Sooty Tern & $\mathrm{R}$ \\
\hline & Sterna sumatrana & Black-naped Tern & $\mathrm{R}$ \\
\hline & Sterna hirundo & Common Tern & M \\
\hline & Chlidonias hybrida & Whiskered Tern & M \\
\hline & Chlidonias leucopterus & White-winged Tern & M \\
\hline Stercorariidae & Stercorarius longicaudus & Long-tailed Jaeger & $\mathrm{P}$ \\
\hline \multirow[t]{4}{*}{ Columbidae } & Caloenas nicobarica & Nicobar Pigeon & $\mathrm{R}$ \\
\hline & Alopecoenas canifrons & Palau Ground Dove & $\mathrm{R}$ \\
\hline & Ptilinopus pelewensis & Palau Fruit Dove & $\mathrm{R}$ \\
\hline & Ducula oceanica & Micronesian Imperial & $\mathrm{R}$ \\
\hline \multirow{7}{*}{ Cuculidae } & Clamator coromandus & $\begin{array}{l}\text { Pigeon } \\
\text { Chestnut-winged Cuckoo }\end{array}$ & V \\
\hline & Urodynamis taitensis & Pacific Long-tailed Cuckoo & V \\
\hline & Scythrops novaehollandiae & Channel-billed Cuckoo & $*$ \\
\hline & Cacomantis variolosus & Brush Cuckoo & $\mathrm{V}$ \\
\hline & Hierococcyx hyperythrus & Rufous Hawk-Cuckoo & V \\
\hline & Cuculus optatus & Oriental Cuckoo & M \\
\hline & Cuculus canorus & Common Cuckoo & V \\
\hline \multirow[t]{2}{*}{ Strigidae } & Pyrroglaux podargina & Palau Owl & $\mathrm{R}$ \\
\hline & Ninox scutulata & Brown Hawk-Owl & V \\
\hline \multirow[t]{2}{*}{ Caprimulgidae } & Caprimulgus jotaka & Gray Nightjar & M \\
\hline & Caprimulgus phalaena & Palau Nightjar & $\mathrm{R}$ \\
\hline \multirow[t]{2}{*}{ Apodidae } & Aerodramus pelewensis & Palau Swiftlet & $\mathrm{R}$ \\
\hline & Hirundapus caudacutus & White-throated Needletail & $\mathrm{V}$ \\
\hline Coraciidae & Eurystomus orientalis & Oriental Dollarbird & M \\
\hline \multirow[t]{4}{*}{ Alcedinidae } & Halcyon coromanda & Ruddy Kingfisher & * \\
\hline & Todiramphus chloris & Collared Kingfisher & $\mathrm{R}$ \\
\hline & $\begin{array}{c}\text { Todiramphus } \\
\text { pelewensis }\end{array}$ & Rusty-capped Kingfisher & $\mathrm{R}$ \\
\hline & Alcedo atthis & Common Kingfisher & $*$ \\
\hline Meropidae & Merops ornatus & Rainbow Bee-eater & $\mathrm{V}$ \\
\hline Falconidae & Falco peregrinus & Peregrine Falcon & (M) \\
\hline Cacatuidae & Cacatua galerita & Sulphur-crested Cockatoo & I \\
\hline Psittaculidae & Eclectus roratus & Eclectus Parrot & I \\
\hline Meliphagidae & Myzomela rubratra & Micronesian Myzomela & $\mathrm{R}$ \\
\hline Artamidae & Artamus leucoryn & $\begin{array}{l}\text { White-breasted } \\
\text { Woodswallow }\end{array}$ & $\mathrm{R}$ \\
\hline Campephagidae & Coracina monacha & Palau Cicadabird & $\mathrm{R}$ \\
\hline Pachycephalidae & Pachycephala tenebrosa & Morningbird & $\mathrm{R}$ \\
\hline Laniidae & Lanius cristatus & Brown Shrike & V \\
\hline Rhipiduridae & Rhipidura lepida & Palau Fantail & $\mathrm{R}$ \\
\hline Monarchidae & Myiagra erythrops & Palau Flycatcher & $\mathrm{R}$ \\
\hline Hirundinidae & Hirundo rustica & Barn Swallow & M \\
\hline Cettiidae & Horornis annae & Palau Bush Warbler & $\mathrm{R}$ \\
\hline Acrocephalidae & Acrocephalus orientalis & Oriental Reed Warbler & $\mathrm{V}$ \\
\hline Locustellidae & Locustella lanceolata & Lanceolated Warbler & V \\
\hline Zosteropidae & $\begin{array}{l}\text { Megazosterops } \\
\text { palauensis }\end{array}$ & Giant White-eye & $\mathrm{R}$ \\
\hline
\end{tabular}


Table 2 (continued).

\begin{tabular}{lllc}
\hline Family & Genus/species $^{a}$ & English name $^{a}$ & Status $^{b}$ \\
\hline \multirow{4}{*}{ Sturnidae } & Zosterops semperi & Citrine White-eye & $\mathrm{R}$ \\
& Zosterops finschii & Dusky White-eye & $\mathrm{R}$ \\
Turdidae & Aplonis opaca & Micronesian Starling & $\mathrm{R}$ \\
& Agropsar philippensis & Chestnut-cheeked Starling & $\mathrm{V}$ \\
& Zoothera dauma & Scaly Thrush & $\mathrm{V}$ \\
Muscicapidae & Turdus obscurus & Eyebrowed Thrush & $\mathrm{V}$ \\
& Muscicapa griseisticta & Gray-streaked Flycatcher & $\mathrm{M}$ \\
& Calliope calliope & Siberian Rubythroat & $\mathrm{V}$ \\
& Ficedula narcissina & Narcissus Flycatcher & $\mathrm{V}$ \\
& Monticola solitarius & Blue Rock Thrush & $\mathrm{V}$ \\
Passeridae & Oenanthe isabellina & Isabelline Wheatear & $*$ \\
Estrildidae & Passer montanus & Eurasian Tree Sparrow & $\mathrm{I}$ \\
& Erythrura trichroa & Blue-faced Parrotfinch & $\mathrm{R}$ \\
Motacillidae & Lonchura atricapilla & Chestnut Munia & $\mathrm{I}$ \\
& Motacilla tschutschensis & Eastern Yellow Wagtail & $\mathrm{M}$ \\
& Motacilla cinerea & Gray Wagtail & $\mathrm{V}$ \\
& Motacilla alba & White Wagtail & $\mathrm{V}$ \\
& Anthus godlewskii & Blyth's Pipit & $\mathrm{V}$ \\
& Anthus cervinus & Red-throated Pipit & $\mathrm{V}$ \\
Emberizidae & Emberiza melanocephala & Black-headed Bunting & $\mathrm{V}$ \\
\hline
\end{tabular}

${ }^{a}$ Endemic species are in boldface. Scientific and English names follow Gill and Donsker (2018) except as noted in text.

${ }^{b}$ Status codes follow Wiles (2005): R, resident species; M, migratory species; S, seabird occasionally sighted near shore inside Palau's barrier reef; $\mathrm{P}$, pelagic species seen only on open seas outside Palau's barrier reef; V, vagrant, I, introduced. Parentheses indicate that a status code is provisional and under review. *, Species newly added to the Palau list in this report.

Palau. A sighting of a bird in alternate plumage on Guam represents the only other confirmed record for Micronesia (Wiles et al. 1993). The Chinese Pond Heron breeds widely in China, Korea, and Japan and winters south to Sumatra and Borneo.

White-breasted Waterhen Amaurornis phoenicurus. P007. One bird (Figure 1d), 30 Oct-3 Nov 2009, Ngaremlengui (Ngermetengel hamlet), Mark Vereen. Buden and Retogral (2010) mentioned this sighting in their report of the first record for Micronesia, based on a breeding population at Woleai Atoll, Yap, Federated States of Micronesia. The committee accepted photos taken by Vereen on 1 Nov 2009 as satisfactory confirmation of the first record for Palau and the second for Micronesia. The species occurs across southern and eastern Asia east to Japan and the Moluccas.

Eurasian Curlew Numenius arquata. P013. One bird (Figures 1e, 1f), 20 Mar-12 Apr 2014, Peleliu (Northern Peleliu Lkes Important Bird Area), Glenn McKinlay. The photograph of the bird in flight (Figure 1e) shows a diagnostic white $\mathrm{V}$ on the rump and lower back; the other photograph (Figure 1f) shows the bird in comparison with a Far Eastern Curlew (N. madagascariensis). This is the first record for Palau. Wiles (2005) listed the Eurasian Curlew as a migrant to Guam and the Northern Marianas.

Broad-billed Sandpiper Calidris falcinellus. P012. Up to three birds (Figure 2a), 27 Sep-27 Oct 2015, Ngiwal (sand flats at Ngirngemelas monument), Milang Eberdong, Glenn McKinlay, Alan Olsen, and Angelina Smaserui-Olsen. The committee accepted this record on the basis of photos showing the diagnostic bill. A male specimen (USNM 384772) taken 21 Sep 1945 on Angaur Island by Baker (1948, 1951) provides the only prior record for Palau and Micronesia. 


\section{FIRST REPORT OF THE PALAU BIRD RECORDS COMMITTEE}

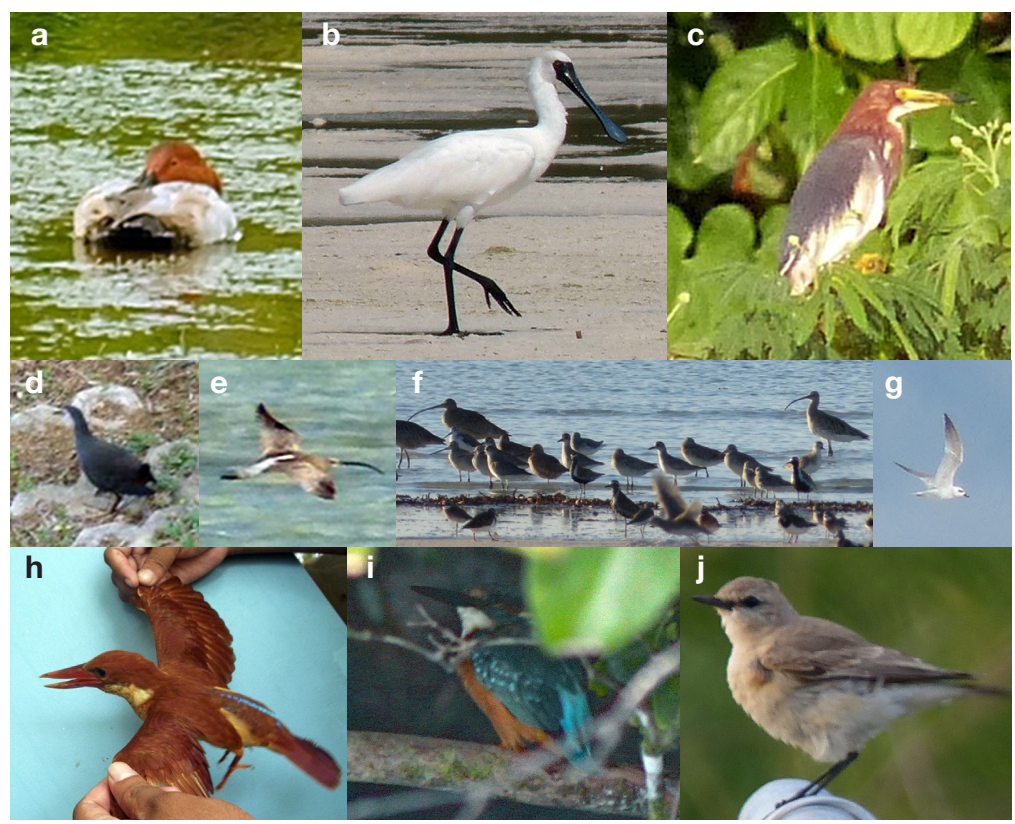

Figure 1. First recorded occurrences in Palau of the (a) Common Pochard (Aythya ferina), Melekeok (Lake Ngardok), 19 Nov 2014; (b) Black-faced Spoonbill (Platalea minor), Peleliu, 12 Dec 2013; (c) Chinese Pond Heron (Ardeola bacchus), Koror, 12 May 2014; (d) White-breasted Waterhen (Amaurornis phoenicurus), Ngaremlengui, 1 Nov 2009; (e) Eurasian Curlew (Numenius arquata), Peleliu, 30 Mar 2014; (f) Eurasian Curlew (right) and Far Eastern Curlew (N. madagascariensis) (left), Peleliu, 30 Mar 2014; (g) Gull-billed Tern (Gelochelidon nilotica), Koror, 14 Dec 2014; (h) Ruddy Kingfisher (Halcyon coromanda), Kayangel, 26 Sep 2011; (i) Common Kingfisher, (Alcedo atthis) Rock Islands Southern Lagoon World Heritage Site, 1 Feb 2012; (j) Isabelline Wheatear (Oenanthe isabellina), Koror, 7 Oct 2014.

Photos by Heather Ketebengang (a), Ron Leidich (i), Glenn McKinlay $(b, c, e, f, g, j)$, Mark Vereen (d), Ality Inawo (h).

Gull-billed Tern Gelochelidon nilotica. P011. One bird (Figure 1g), 14 Dec 2014, Peleliu (Northern Peleliu Lkes Important Bird Area), Glenn McKinlay. The bird's adult basic plumage (with no signs of breeding plumage in December) and bill shape (with prominent gonydeal angle) point to the southeast Asian subspecies G. n. affinis rather than the Australian G. n. macrotarsa. This is the first record for Palau. Wiles (2005) listed this species as vagrant to Guam.

Chestnut-winged Cuckoo Clamator coromandus. P018. One bird (Figure 2b), 1 Mar 2013, Kayangel, Vivian Eledui. Photos of the bird in hand show the distinctive black upperparts and crest, white hind collar, rufous wings and throat, and white underparts. The only previous record for Palau is of a specimen (USNM 526212) collected 4 Jun 1967 in Koror (Owen 1977a) and verified by C. Milensky (pers. comm.) as a Chestnut-winged Cuckoo. The species is widespread in south and southeast Asia and migratory at least in China. 


\section{FIRST REPORT OF THE PALAU BIRD RECORDS COMMITTEE}

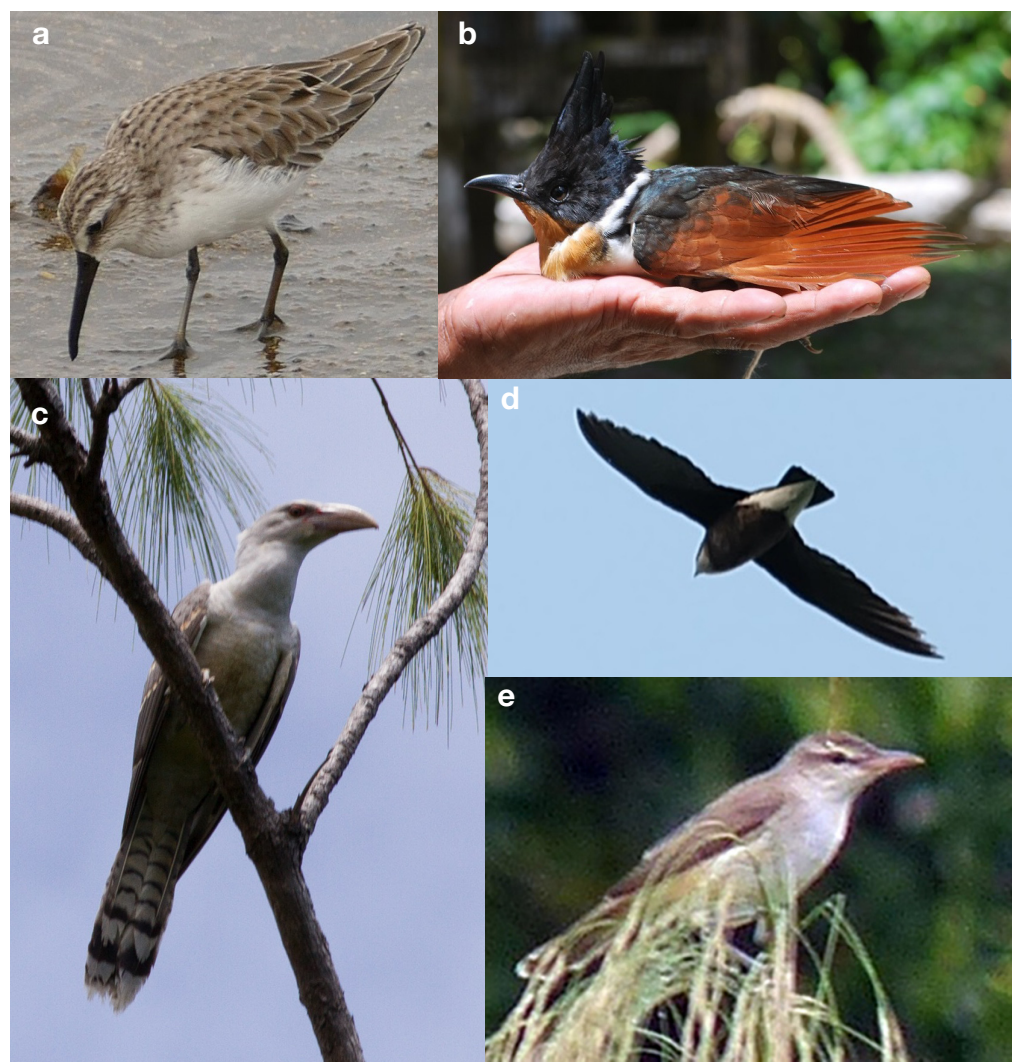

Figure 2. Second recorded occurrences in Palau of the (a) Broad-billed Sandpiper (Calidris falcinellus), Ngiwal, 27 Oct 2015; (b) Chestnut-winged Cuckoo (Clamator coromandus), Kayangel, 1 March 2013; (c) Channel-billed Cuckoo (Scythrops novaehollandiae), Angaur, 3 Nov 2013; (d) White-throated Needletail (Hirundapus caudacutus), Kayangel, 21 Oct 2015; (e) Oriental Reed Warbler (Acrocephalus orientalis), Koror, 10 Dec 2013.

Photos by Alan Olsen (a), Vivian Eledui (b), Joel Miles (c), Heather Ketebengang (d), Glenn McKinlay (e).

Channel-billed Cuckoo Scythrops novaehollandiae P005a. One bird, 14 Jul 2011, Koror, Heather Ketebengang. The committee accepted this first record on the basis of Ketebengang's photo and description of the bird's distinctive morphology. The committee also accepted a second record (P005b) supported by a higher-quality photo (Figure 2c) of an immature bird with a diagnostic massive bill, red eye, buff areas on the wing and underparts, and a long tail with black and white tip, found on Angaur by Joel Miles, 3 Nov 2013. This Australasian species, migratory in the southern part of its range, has not been previously reported from Micronesia.

White-throated Needletail Hirundapus caudacutus. P017. One bird (Figure 2d), 21 Oct 2015, Kayangel, Heather Ketebengang. Photos of the bird in flight show a 
diagnostic combination of white chin, dark underbody, and white undertail. The only previous record for Palau was of four birds sighted on 13 Apr 2004 on Bloody Nose Ridge, Peleliu (Pratt et al. 2010; Table 1).

Ruddy Kingfisher Halcyon coromanda. P008. Two birds (Figure 1h), 26 Sep 2011, Kayangel, Ality Inawo. The photos clearly show the ruddy plumage with a diagnostic blue dorsal streak. This species has not been previously reported from Micronesia. Its patchy distribution includes breeding in Japan and Korea and wintering in the Philippines.

Common Kingfisher Alcedo atthis. P004. One bird (Figure 1i), 1 Feb 2012, Koror (Rock Islands Southern Lagoon World Heritage Site), Ron Leidich. The photo shows the characteristic white chin and facial marks, reddish brown underparts, and bright blue dorsum. This is the first unequivocal record of this species from Palau. A sighting on Guam is the only other record for Micronesia (Wiles et al. 1993). The migratory subspecies bengalensis breeds as far east as the Kuril Islands and winters regularly east to the Philippines and Moluccas; resident subspecies occur as far east as the Solomon Islands.

Oriental Reed Warbler Acrocephalus orientalis. P014. Two birds (Figure 2e), 9 Dec 2013 to 18 Mar 2014, Koror (Malakal sewage treatment plant), Glenn McKinlay. The committee accepted this record on the basis of photos showing a plain warbler with a combination of bill, body, and tail proportions and body plumage typical of the Oriental Reed Warbler. A white supercilium and dark eye stripe, both distinctly extending well behind the eye, and white-tipped tail feathers, distinguish the bird from congeners in its range. McKinlay further informed the committee that the bird's call recorded on video matched that of the Oriental Reed Warbler, including a sharp harsh single "chkt," rapid sequences "cht-cht-cht-cht," and longer warbling sequences. The only other record of this species for Palau is of a pair of birds photographed at the same location on 11 Apr 2007 (Pratt et al. 2010; Table 1). The Oriental Reed Warbler breeds from central China northeast to Sakhalin and winters in southeast Asia east regularly to the Philippines and Moluccas, rarely to New Guinea.

Isabelline Wheatear Oenanthe isabellina. P009. One bird (Figure 1j), 7 Oct 2014, Koror (Malakal sewage treatment plant), Glenn McKinlay. The photos show several diagnostic features, including a black alula that contrasts with the rest of the wing, wings not contrasting with the back, and an eyebrow white in front of the eye. This species has not been previously reported from Micronesia. Its normal range is central Asia to Arabia and north Africa, with vagrants recorded as far east as Australia, Japan, and the Korean peninsula (Shimba 2007, Christidis and Boles 2008, Gill and Donsker 2018).

\section{OTHER COMMITTEE ACTIONS}

The committee reviewed three additional items.

Chinese Sparrowhawk Accipiter soloensis. P016. One bird, 17 Sep 2015, Kayangel, Ality Inawo. The committee accepted this report as a valid but not particularly unusual observation because we consider this species the raptor that visits Palau most frequently (Wiles et al. 2000, Pratt and Etpison 2008). Because of uncertainty concerning the status of various raptors as migrant or vagrant in Palau (cf. Wiles 2005, Pratt and Etpison 2008), the committee reviews all reports of raptors to help clarify the status of each species.

Latham's Snipe Gallinago hardwickii. P015. This report of a possible first record was placed in abeyance pending receipt of additional information. Photos reviewed by the committee were inconclusive with regard to identification characteristics, especially 
the number and shapes of the rectrices, which help distinguish G. hardwickii from other species of Gallinago known to occur in Palau.

Blyth's Pipit Anthus godlewskii. P002. One bird, 23 Apr 2005, Koror (Malakal sewage treatment plant), originally reported as Richard's Pipit (A. richardi; VanderWerf et al. 2006). The original photos were re-examined by Lees and VanderWerf (2011) and the identification was corrected to A. godlewskii. This correction is reflected in Tables 1 and 2 .

\section{THE PALAU ISLANDS BIRD LIST}

As of December 2016, a total of 168 species are known to have occurred in Palau (Table 2): 158 species from the historical list and 10 new species accepted by the committee. Table 2 represents the official Palau Islands bird list of the PBRC. English and scientific names follow Gill and Donsker (2018) except the English name Rufous Night Heron is applied to Nycticorax caledonicus to reflect local usage for the resident subspecies $N$. c. pelewensis, and the spelling of the species name of the White-breasted Woodswallow is emended from Artamus leucorynchus to A. leucocoryn to be consistent with article 32.5.1 of the International Code of Zoological Nomenclature (David and Dickinson 2014).

\section{ACKNOWLEDGMENTS}

We are grateful for the support of the Belau National Museum and the Palau Conservation Society and for funding from the Marisla Foundation via the Global Greengrants Fund, Global Environment Facility Small Grants Program through UNDP, and Global Environment Facility Palau STAR Project through UN Environment (UNEP). We thank Daniel D. Gibson, Gary Rosenberg, and Kimball Garrett for their helpful in-depth reviews of our manuscript, Christopher Milensky of the U.S. National Museum of Natural History for assistance with the confirmation of the Chestnut-winged Cuckoo specimen, David Ramarui for organizing the database for this report, and the citizen scientists who contributed their observations and photos: Vivian Eledui, Ality Inawo, Joel Miles, Angelina Smaserui-Olsen and Mark Vereen. The PBRC welcomes noteworthy reports of bird observations sent to palaubirdrecords@gmail.com.

\section{LITERATURE CITED}

Baker, R. H. 1948. Report of collections of birds made by United States Naval Medical Research Unit No. 2 in the Pacific war area. Smithson. Misc. Coll. 107:1-74.

Baker, R. H. 1951. The avifauna of Micronesia, its origin, evolution and distribution. Univ. Kansas Publ., Mus. Nat. Hist. 3:1-359.

BirdLife International. 2018. Important Bird Areas factsheet: Northern Peleliu Lkes (sandflats); www.birdlife.org (5 Jun 2018).

Bruyns, W. F. J. M. 1964. Birds seen during west to east trans-Pacific crossing along equatorial counter-current around latitude $7^{\circ} \mathrm{N}$. in the autumn of 1960 . Sea Swallow 17:57-66.

Buden, D. W., and Retogral, S. 2010. Range expansion of the White-breasted Waterhen (Amaurornis phoenicurus) into Micronesia. Wilson J. Ornithol. 122:784-788.

Christidis, L., and Boles, W. E. 2008. Systematics and Taxonomy of Australian Birds. CSIRO Publishing, Collingwood, Victoria, Australia.

David, N., and Dickinson, E. C. 2014. Appendix 8: Changes in the spellings of scientific names, in The Howard \& Moore Complete Checklist of the Birds of the 
World (E. C. Dickinson and J. V. Remsen, eds.), vol. 2. Aves Press, Eastbourne, England.

Dixon, K. L., and Starrett, W. C. 1952. Offshore observations of tropical sea birds in the western Pacific. Auk 69:266-272.

Engbring, J. 1983. Avifauna of the southwest islands of Palau. Atoll Res. Bull. 267.

Engbring, J. 1988. Field Guide to the Birds of Palau. Conservation Office, Koror, Palau.

Engbring, J., and Owen, R. P. 1981. New bird records for Micronesia. Micronesica 17:186-192.

Finsch, O. 1875. Zur ornithologie der Süsee-Inseln. I. Die Vögel der Palau-Gruppe. J. Mus. Godeffroy 8:133-183.

Gill, F., and Donsker, D. (eds). 2018. IOC world bird list, version 8.1; www.worldbirdnames.org (1 Mar 2018).

Hachisuka, M. U., Kuroda, N., Takatsukasa, N., Uchida, S., and Yamashina, Y. 1942. A Hand-list of Japanese Birds, rev. ed. Ornithol. Soc. Japan, Tokyo.

Hartlaub, G. 1868. On a collection of birds from some lesser-known localities in the western Pacific. Proc. Zool. Soc. London for 1867, pp. 828-832.

Hartlaub, G., and Finsch, O. 1868a. On a collection of birds from the Pelew Islands. Proc. Zool. Soc. London for 1868, pp. 4-9.

Hartlaub, G., and Finsch, O. 1868b. Additional notes on the ornithology of the Pelew Islands. Proc. Zool. Soc. London for 1868, pp. 116-118.

Hartlaub, G., and Finsch, O. 1872. On a fourth collection of birds from the Pelew and Mackenzie Islands. Proc. Zool. Soc. London for 1872, pp. 87-114.

IUCN. 2017. The IUCN Red List of threatened species, version 2017-3; www. iucnredlist.org (7 Mar 2018).

Kuroda, N. 1922a. Descriptions of two new forms of birds from Pelew Islands, in Birds of Micronesia (T. Momiyama, ed.), part I, pp. 25-30. Ornithol. Soc. Japan, Tokyo.

Kuroda, N. 1922b. A list of the birds of Micronesia Group, exclusive of Magalhaes, Gilbert and Ellis islands, in Birds of Micronesia (T. Momiyama, ed.), part I, pp. 31-78. Ornithol. Soc. Japan, Tokyo.

Lees, A. C., and VanderWerf, E. A. 2011. First record of Blyth's Pipit, Anthus godlewskii, for Micronesia. Bull. Br. Ornithol. Club 131:121-216.

Marshall, J. T. 1951. The endemic avifauna of Saipan, Tinian, Guam and Palau. Condor 51:200-221.

Mayr, E. 1945. Birds of the Southwest Pacific. MacMillan, New York.

McKinlay, G. 2015. First record of Black-faced Spoonbill Platalea minor for Palau: An identification challenge. BirdingASIA 23:135-136.

Momiyama, T. (ed). 1922. Birds of Micronesia, part I. Ornithol. Soc. Japan, Tokyo.

Owen, R. P. 1977a. New bird records for Micronesia and major island groups in Micronesia. Micronesica 13:57-63.

Owen, R. P. 1977b. A checklist of the birds of Micronesia. Micronesica 13:65-81.

Pratt, H. D., and Etpison, M. T. 2008. Birds and Bats of Palau. Mutual Publ., Honolulu.

Pratt, H. D., Engbring, J., Bruner, P. L., and Berrett, D. G. 1980. Notes on the taxonomy, natural history, and status of the resident birds of Palau. Condor 82:117-131.

Pratt, H. D., Bruner, P. L., and Berrett, D. G. 1987. A Field Guide to the Birds of Hawaii and the Tropical Pacific. Princeton Univ. Press, Princeton, NJ.

Pratt, H. D., Falanruw, M., Etpison, M. T., Olsen, A., Buden, D. W., Clements, P., Gupta, A., Ketebengang, H., Yalap, Y., Herter, D. R., Klauber, D., Pisano, P., Vice, D. S., and Wiles, G. J. 2010. Noteworthy bird observations from the Caroline and Marshall Islands 1988-2009, including five new records for Micronesia. W. Birds 41:70-101. 


\section{FIRST REPORT OF THE PALAU BIRD RECORDS COMMITTEE}

Pyle, P., and Engbring, J. 1985. Checklist of the birds of Micronesia. 'Elepaio 46:57-68.

Rauzon, M. J. 1988. Red-kneed Dotterel in Belau: First record for Micronesia. 'Elepaio 48:56-57.

Ripley, S. D. 1948. First record of Anhingidae in Micronesia. Auk 65:454-455.

Ripley, S. D. 1951. Migrants and introduced species in the Palau Archipelago. Condor 53:299-300.

Shimba, T. 2007. A Photographic Guide to the Birds of Japan and North-east Asia. Yale Univ. Press, New Haven, CT.

VanderWerf, E. A., Wiles, G. J., Marshall, A. P., and Knecht, M. 2006. Observations of migrants and other birds in Palau, April-May 2006, including the first Micronesian record of a Richard's Pipit. Micronesica 39:11-29.

Wiles, G. J. 2005. A checklist of the birds and mammals of Micronesia. Micronesica 38:141-189.

Wiles, G. J., and Conry, P. J. 1990. Terrestrial vertebrates of the Ngerukewid Islands wildlife preserve. Micronesica 23:41-66.

Wiles, G. J., Beck, R. E. Jr., Aguon, C. F., and Orcutt, K. D. 1993. Recent bird records for the southern Mariana Islands, with notes on a colony of Black Noddies on Cocos Island, Guam. Micronesica 26:199-215.

Wiles, G. J., Worthington, D. J., Beck, R. F., Pratt, H. D., Aguon, C. F., and Pyle, R. L. 2000. Noteworthy bird records for Micronesia, with a summary of raptor sightings in the Mariana Islands, 1988-1999. Micronesica 32:257-284.

Wiles, G. J., Johnson, N. C., de Cruz, J. B., Dutson, G., Camacho, V. A., Kepler, A. K., Vice, D. S., Garrett, K. L., Kessler, C. C., and Pratt, H. D. 2004. New and noteworthy bird records for Micronesia, 1986-2003. Micronesica 37:69-96.

Accepted 5 June 2018 